\title{
Nuclear Constraints on the Weak Nucleon-Nucleon Interaction
}

\author{
W. C. Haxton \\ Institute for Nuclear Theory and Department of Physics, \\ University of Washington, Seattle, WA 98195, USA \\ Email: haxton@phys.washington.edu
}

\begin{abstract}
I discuss the current status of efforts to constrain the strangeness-conserving weak hadronic interaction, which can be isolated in nuclear systems because of the associated parity violation.
\end{abstract}

PACS numbers:

Keywords: Weak interactions, parity nonconservation, anapole moments.

\section{PARITY NONCONSERVATION IN THE NN SYSTEM}

In this talk I will discuss the weak nucleon-nucleon (NN) interaction: the experiments that have been done, the strategies theorists have developed to interpret measurements, and the puzzles that remain to be resolved.

While the weak interaction can be observed in flavor-changing hadronic decays, the neutral current contribution to such decays is suppressed by the GIM mechanism and thus unobservable. The NN and nuclear systems are thus the only practical laboratories for studying the hadronic weak interaction in all of its aspects 1, 2,

As the weak contribution to the NN interaction is many orders of magnitude smaller than the strong and electromagnetic contributions (the suppression relative to the strong interaction is $\sim 4 \pi G m_{\pi}^{2} / g_{\pi N N}^{2} \sim 10^{-7}$ ), parity violation must be exploited to isolate weak observables. The most common observables are pseudoscalars arising from the interference of the weak and strong/electromagnetic interactions, e.g., the circular polarization of $\gamma$ rays emitted from an unpolarized excited nuclear state, or the $\gamma$ ray asymmetry if the nuclear state can be polarized. The observable depends on an interference between parity-conserving (PC) and parity-non-conserving (PNC) amplitudes, and the weak interaction appears linearly. Alternatively, there are processes, such as the $\alpha$ decay of an unnatural-parity nuclear state to a $0^{+}$final state, where the amplitude is entirely weak. Such observables are proportional to the squares of weak matrix elements, and thus are not associated with a pseudoscalar.

The range of the underlying weak interaction, mediated by W and $\mathrm{Z}$ exchange, is $\sim 0.002 \mathrm{fm}$, much smaller than the radius of the nucleon. For this reason the nuclear weak force is often modeled as a series of meson exchanges, with one nucleon vertex strong and with the second vertex containing the weak physics, as depicted in Fig. ??. The resulting isospin of the weak meson-nucleon coupling is related to the underlying currents in an interesting way. The hadronic weak interaction has the low-energy current-current form

$$
L^{e f f}=\frac{G}{\sqrt{2}}\left[J_{W}^{\dagger} J_{W}+J_{Z}^{\dagger} J_{Z}\right]+\text { h.c. }
$$

where the charge-changing current is the sum of $\Delta \mathrm{I}=1 \Delta \mathrm{S}=0$ and Cabibbo-suppressed $\Delta \mathrm{I}=1 / 2 \Delta \mathrm{S}=-1$ terms,

$$
J_{W}=\cos \theta_{C} J_{W}^{\Delta S=0}+\sin \theta_{C} J_{W}^{\Delta S=-1} .
$$

Consequently the $\Delta \mathrm{S}=0$ interaction has the form

$$
L_{\Delta S=0}^{e f f}=\frac{G}{\sqrt{2}}\left[\cos ^{2} \theta_{C} J_{W}^{0 \dagger} J_{W}^{0}+\sin ^{2} \theta_{C} J_{W}^{1 \dagger} J_{W}^{1}+J_{Z}^{\dagger} J_{Z}\right]
$$

where the first term, a symmetric product of $\Delta \mathrm{I}=1$ currents, has $\Delta \mathrm{I}=0,2$, while the second term, a symmetric product of $\Delta \mathrm{I}=1 / 2$ currents, is $\Delta \mathrm{I}=1$ but Cabibbo suppressed. Consequently a $\Delta \mathrm{I}=1$ PNC meson-nucleon vertex should be dominated by the neutral current term - a term not accessible in strangeness-changing processes. One could isolate this term by an isospin analysis of a complete set of PNC NN observables.

\section{S-P AMPLITUDES AND MESON-EXCHANGE POTENTIALS}

There are several ways to describe low-energy PNC NN interactions. Perhaps the simplest representation, the Danilov amplitudes, is an S-P partial wave description appropriate in the low-momentum limit. Table $\mathbb{I}$ lists the five partial waves. The coefficients multiplying these amplitudes can be treated as free parameters, to be determined 


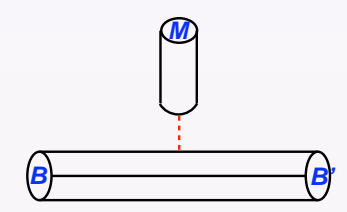

(a) Factorization

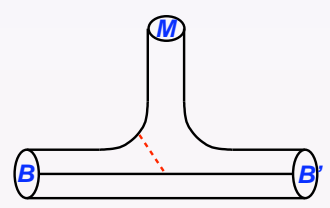

(b) Quark Model

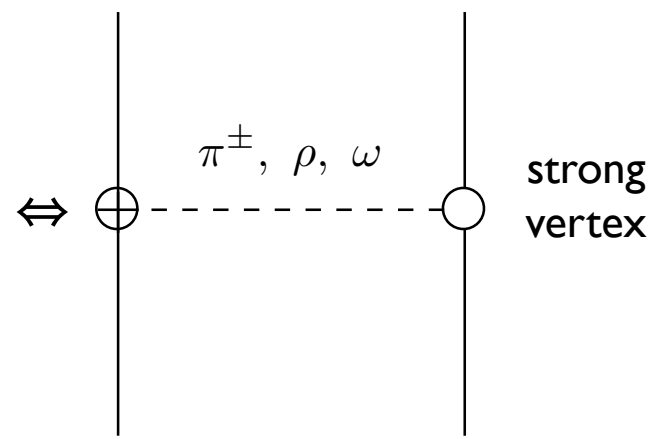

(c) Sum Rule

FIG. 1: A single-boson-exchange contribution to $V_{P N C}$ contains one weak vertex (left) and one strong one (right). The weak vertex is decomposed into the quark-level terms that DDH estimated, using the standard model in combination with techniques such as factorization, the quark model, and sum rules.

TABLE I: S-P weak PNC amplitudes and the corresponding meson-exchanges [1].

\begin{tabular}{cccccccc}
\hline \hline Transition & $\mathrm{I} \leftrightarrow \mathrm{I}^{\prime}$ & $\Delta$ & $\mathrm{I}$ & $\mathrm{n}-\mathrm{n}$ & $\mathrm{n}-\mathrm{p}$ & $\mathrm{p}-\mathrm{p}$ & meson exchanges \\
\hline${ }^{3} S_{1} \leftrightarrow{ }^{1} P_{1}$ & $0 \leftrightarrow \leftrightarrow$ & 0 & & & $\mathrm{x}$ & & $\rho, \omega$ \\
${ }^{1} S_{0} \leftrightarrow{ }^{3} P_{0}$ & $1 \leftrightarrow 1$ & 0 & $\mathrm{x}$ & $\mathrm{x}$ & $\mathrm{x}$ & $\rho, \omega$ \\
& & 1 & $\mathrm{x}$ & & $\mathrm{x}$ & $\rho, \omega$ \\
& & 2 & $\mathrm{x}$ & $\mathrm{x}$ & $\mathrm{x}$ & $\rho$ \\
& & & & & & & \\
${ }^{3} S_{1} \leftrightarrow{ }^{3} P_{1}$ & $0 \leftrightarrow$ & 1 & & $\mathrm{x}$ & & $\pi^{ \pm}, \rho, \omega$ \\
\hline \hline
\end{tabular}

from experiments. Once these are fixed, other low-energy PNC observables could be predicted, in virtually a modelindependent way.

A second approach expresses the interaction as a set of single meson exchanges (see Fig. ??), in analogy with traditional meson-exchange treatments of the strong force, but with one of the strong vertices replaced by a weak one containing the short-range W,Z physics. The possible exchanges are constrained by symmetries, e.g., Barton's theorem excludes on-shell couplings to neutral scalar mesons. If one includes $\rho, \omega$, and $\pi^{ \pm}$exchanges, one has enough freedom to reproduce the five Danilov amplitudes and to model the long-range pion contribution important to higher partial waves.

Much of the work that has been done in the field uses a potential developed by Donoghue, Desplanques, and Holstein $(\mathrm{DDH})$ 4

$$
\begin{aligned}
2 M V^{P N C}(\vec{r}) & =\frac{g_{\pi N N} f_{\pi}}{\sqrt{2}} \tau_{\times}^{z} \vec{\sigma}_{+} \cdot \vec{u}_{\pi} \\
& -g_{\rho}\left[h_{\rho}^{0} \vec{\tau}_{1} \cdot \vec{\tau}_{2}+h_{\rho}^{1} \tau_{+}^{z}+h_{\rho}^{2} \tau^{z z}\right]\left[\left(1+\mu_{v}\right) \vec{\sigma}_{\times} \cdot \vec{u}_{\rho}+\vec{\sigma}_{-} \cdot \vec{v}_{\rho}\right] \\
& -g_{\omega}\left[h_{\omega}^{0}+h_{\omega}^{1} \tau_{+}^{z}\right]\left[\left(1+\mu_{s}\right) \vec{\sigma}_{\times} \cdot \vec{u}_{\omega}+\vec{\sigma}_{-} \cdot \vec{v}_{\omega}\right] \\
& -\tau_{-}^{z} \vec{\sigma}_{+} \cdot\left[g_{\omega} h_{\omega}^{1} \vec{v}_{\omega}-g_{\rho} h_{\rho}^{1} \vec{v}_{\rho}\right]-\tau_{\times}^{z} g_{\rho} h_{\rho}^{\prime 1} \vec{\sigma}_{+} \cdot \vec{u}_{\rho}
\end{aligned}
$$

where

$$
\begin{gathered}
\vec{\sigma}_{\times} \equiv i \vec{\sigma}_{1} \times \vec{\sigma}_{2} \quad \vec{\sigma}_{+} \equiv \frac{1}{2}\left[\vec{\sigma}_{1}+\vec{\sigma}_{2}\right] \quad \vec{\sigma}_{-} \equiv \vec{\sigma}_{1}-\vec{\sigma}_{2} \\
\tau^{z z} \equiv \frac{1}{2 \sqrt{6}}\left[3 \tau_{1}^{z} \tau_{2}^{z}-\vec{\tau}_{1} \cdot \vec{\tau}_{2}\right] \\
\vec{u}(\vec{r}) \equiv\left[\vec{p}, e^{-m r} / 4 \pi r\right] \quad \vec{v}(\vec{r}) \equiv\left\{\vec{p}, e^{-m r} / 4 \pi r\right\} \quad \vec{p} \equiv \vec{p}_{1}-\vec{p}_{2} .
\end{gathered}
$$

The $g_{\pi N N}, g_{\rho}$, and $g_{\omega}\left(f_{\pi}, h_{\rho}\right.$, and $\left.h_{\omega}\right)$ are the strong (weak) $\pi^{ \pm}, \rho$, and $\omega$ couplings. As noted previously, the limit $m_{\rho}, m_{\omega} \rightarrow \infty$ maps the short-range part of this potential onto the five Danilov amplitudes. (As there are six shortrange $\rho$ and $\omega$ couplings, there is a transformation among these couplings that leaves the S-P amplitudes unchanged [1.) The estimated parameter ranges and best values recommended by DDH are shown in Table II , along with several 
TABLE II: Recommended ranges and best values for the DDH potential, along with three other parameterizations. From [2].

\begin{tabular}{cccccc}
\hline \hline Coupling $\left(\times 10^{-7}\right)$ & DDH Range [4] & Best [4] & DZ [5] & FCDH [] & KM [7] \\
\hline$f_{\pi}$ & $0.0 \leftrightarrow 11.4$ & 4.6 & 1.1 & 2.7 & 0.2 \\
$h_{\rho}^{0}$ & $-30.8 \leftrightarrow 11.4$ & -11.4 & -8.4 & -3.8 & -3.7 \\
$h_{\rho}^{1}$ & $-0.4 \leftrightarrow 0.0$ & -0.2 & 0.4 & -0.4 & -0.1 \\
$h_{\rho}^{2}$ & $-11.0 \leftrightarrow-7.6$ & -9.5 & -6.8 & -6.8 & -3.3 \\
$h_{\omega}^{0}$ & $-10.3 \leftrightarrow 5.7$ & -1.9 & -3.8 & -4.9 & -6.2 \\
$h_{\omega}^{1}$ & $-1.9 \leftrightarrow-0.8$ & -1.1 & -2.3 & -2.3 & -1.0 \\
$h_{\rho}^{\prime}{ }_{1}^{0}$ & & 0.0 & & & -2.2 \\
\hline \hline
\end{tabular}
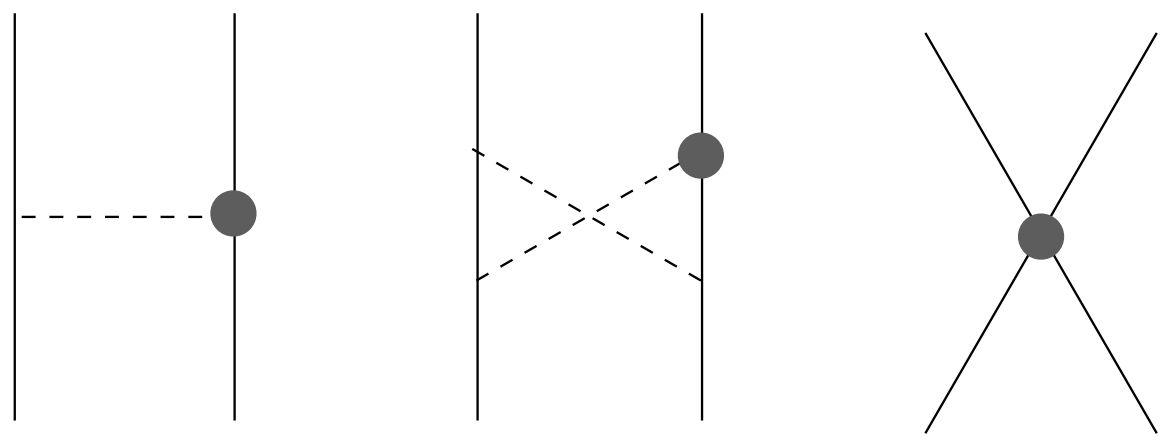

FIG. 2: Contributions in a chiral perturbation theory expansion of $V_{P N C}$ including long-range pion exchange, the intermediaterange contribution from crossed pions, and S-P contact interactions [8].

other parameterizations of this potential. Such a meson-exchange treatment, by providing a model for P-D and other higher partial-waves, presumably has some validity when extended to higher momenta: analogous treatments of the strong potential are quite successful in describing intermediate-range NN interactions.

A third approach, developed recently, is a fully systematic expansion in chiral perturbation theory [8] in terms of $m_{\pi} / \Lambda_{\chi S B}$. This allows for a consistent treatment of iterated pions, helping to define the PNC potential at intermediate ranges: the contributions, illustrated in Fig. 2, include such terms, long-range single $\pi^{ \pm}$exchange, and five contact interactions. While ten parameters arise in this approach in leading order, only five are independent in the limit of low momentum [8, 9].

\section{EXPERIMENTAL CONSTRAINTS}

The goal of the field has been to determine the weak meson-nucleon coupling strengths by fitting to experiment. If the nonperturbative strong interaction physics associated with the meson-nucleon vertices can be computed, one would then be able to connect these vertices with the underlying elementary couplings of the standard model. Ideally one would make a complete set of measurements in the NN system. However, in most cases the required sensitivity is difficult to achieve. The longitudinal analyzing power for $\vec{p}+p$ has been measured at Bonn [10] and SIN [11],

$$
\begin{aligned}
A_{L}^{\vec{p}+p}(13.6 \mathrm{MeV}) & =(-0.93 \pm 0.21) \times 10^{-7} \quad(\text { Bonn }) \\
A_{L}^{\vec{p}+p}(45 \mathrm{MeV}) & =(-1.57 \pm 0.23) \times 10^{-7} \quad(\mathrm{SIN}),
\end{aligned}
$$

constraining the ${ }^{1} S_{0}-{ }^{3} P_{0} \Delta \mathrm{I}=0,1,2$ amplitudes. The circular polarization of the $\gamma \mathrm{s}$ produced in $n+p$ radiative capture has also been measured [12,

$$
P_{\gamma}(n+p \rightarrow d+\gamma)=(1.8 \pm 1.8) \times 10^{-7} .
$$

$P_{\gamma}$ depends on the ${ }^{1} S_{0}-{ }^{3} P_{0} \Delta \mathrm{I}=0,2$ and ${ }^{3} S_{1}-{ }^{1} P_{1} \Delta \mathrm{I}=1$ amplitudes. Finally, there is a upper bound on the $\gamma$-ray asymmetry in radiative capture [13]

$$
A_{\gamma}(\vec{n}+p \rightarrow d+\gamma)=(0.6 \pm 2.1) \times 10^{-7}
$$




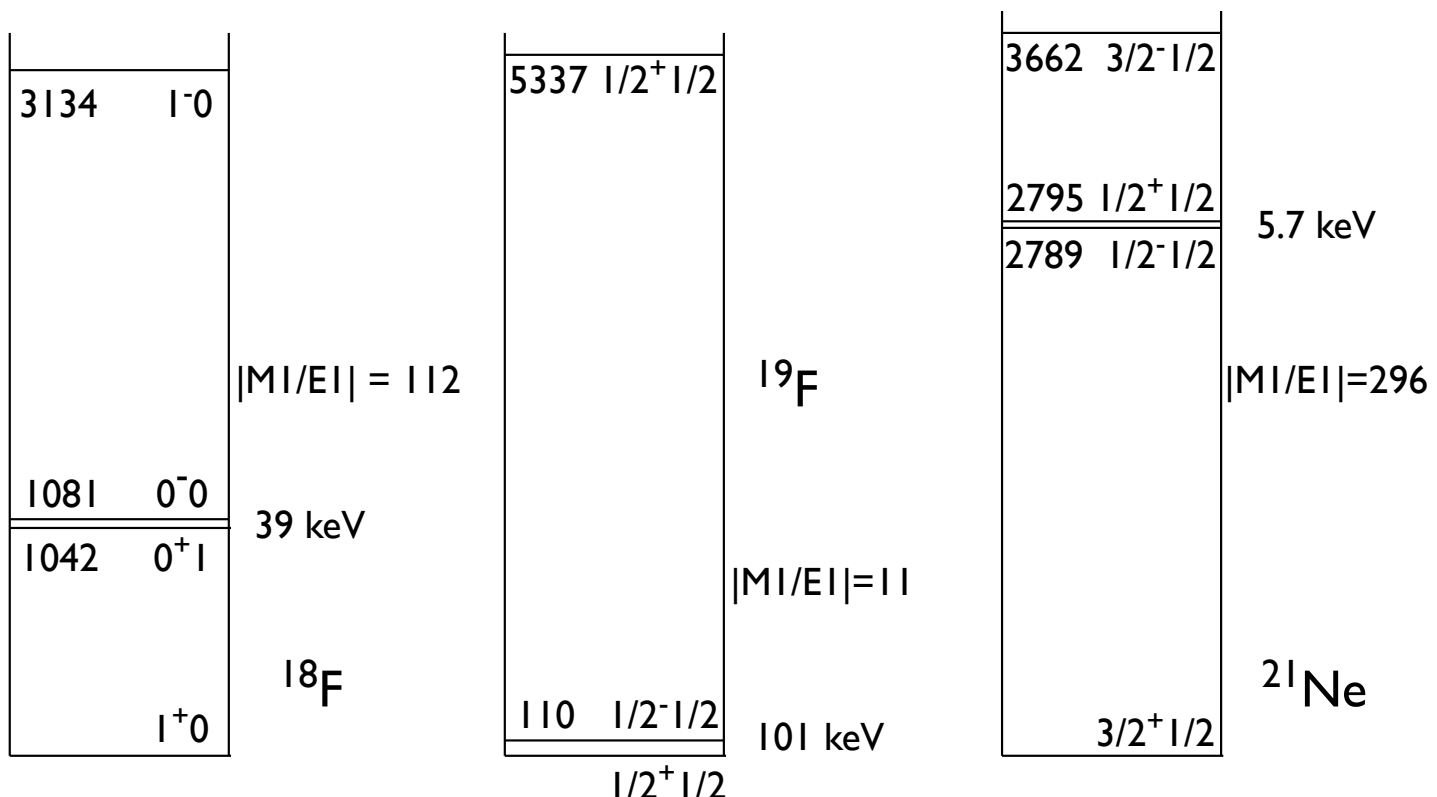

FIG. 3: Three sd-shell two-level nuclear systems in which PNC observables are enhanced.

$A_{\gamma}$ depends on the ${ }^{3} S_{1}-{ }^{3} P_{1} \Delta \mathrm{I}=1$ amplitude. A program has begun at LANSCE and will continue at the SNS to improve this result, with a factor of 20 the long-term goal. There are also plans to measure the spin rotation of polarized neutrons passing through parahydrogen at the SNS [14.

As there are quasi-exact methods for treating few-body nuclei, PNC observables in such systems can also be interpreted reliably. The analyzing power for polarized protons scattering on ${ }^{4} \mathrm{He}$ has been measured [15]

$$
A_{L}^{\vec{p}+{ }^{4} \mathrm{He}}(46 \mathrm{MeV})=(-3.3 \pm 0.9) \times 10^{-7} \quad(\mathrm{SIN}) .
$$

This "odd proton" observable depends on a combination of isovector and isoscalar couplings quite similar to that tested in ${ }^{19} \mathrm{~F}$, discussed below. There are also two bounds of interest,

$$
\begin{aligned}
\frac{d}{d z} \phi_{n}^{\vec{n}+\alpha}(\text { thermal }) & =(8 \pm 14) \times 10^{-7} \mathrm{rad} / \mathrm{m} \quad(\mathrm{NIST} \rightarrow \mathrm{SNS}) \\
A_{L}^{\vec{p}+d}(15 \mathrm{MeV}) & =(-0.35 \pm 0.85)(\mathrm{LANL}) .
\end{aligned}
$$

The NIST effort on the neutron spin rotation is in progress [16. There are plans to continue the work at the SNS.

Measurements in complex nuclei comprise the third class of experiments. One advantage of nuclear experiments is the opportunity, because of level degeneracies and favorable $\mathrm{PNC} / \mathrm{PC}$ matrix element ratios, to significantly enhance the size of PNC observables. There are nuclear PNC effects of $\sim 10 \%$, in contrast to the $10^{-7}$ characteristic of the NN system. One can also use isospin and other nuclear quantum numbers as a filter, isolating specific components of the PNC interaction. The disadvantage of such systems is wave function complexity, which complicates the extraction of coupling strengths from the observables.

Figure 3 shows three nuclei of interest, the parity doublets in ${ }^{18} \mathrm{~F},{ }^{19} \mathrm{~F}$, and ${ }^{21} \mathrm{Ne}$. These are effectively two-level systems because the small parity-doublet splittings $(39,101$, and $5.7 \mathrm{keV})$ make doublet mixing much more important than mixing with distant states. The theoretical challenge is to identify methods for calculating two-level mixing accurately.

${ }^{18} \mathrm{~F}$ is an interesting case for illustrating both the sources of PNC enhancement and the nuclear structure analysis. The circular polarization of the $\gamma$ ray emitted in the decay of the $1081 \mathrm{keV} 0^{-} 0$ to the $1^{+} 0$ ground state is given by

$$
P_{\gamma}(1081 \mathrm{keV})=2 \operatorname{Re}\left[\frac{\left\langle 0^{+} 1\left|V_{P N C}\right| 0^{-} 0\right\rangle}{39 \mathrm{keV}} \frac{\left\langle 1^{+} 0(\text { g.s. })|M 1| 0^{+} 1\right\rangle}{\left\langle 1^{+} 0(\text { g.s. })|E 1| 0^{-} 0\right\rangle}\right]
$$

As the typical scale of PNC nuclear mixing matrix elements is $\sim 1 \mathrm{eV}$, the first ratio in Eq. 11 is $\sim 10^{-5}$. The second term is the ratio of a PNC M1 transition to the normal PC E1 transition. Both transition strengths are known 

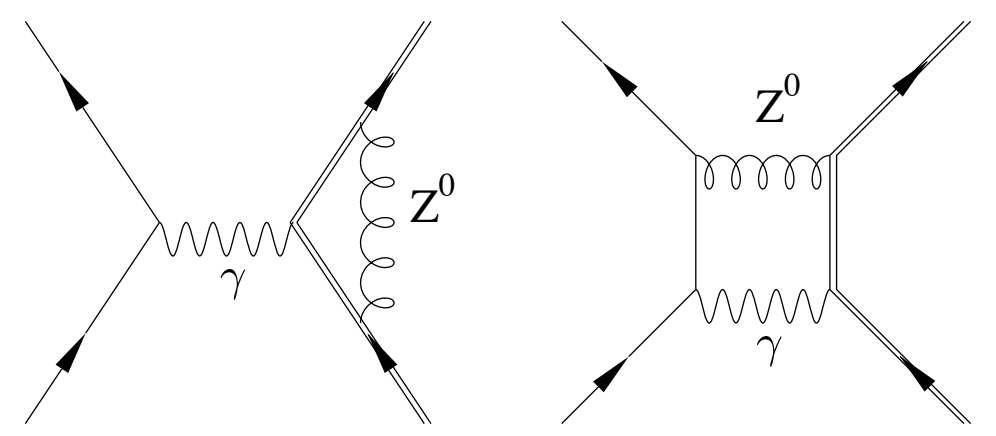

FIG. 4: Weak radiative corrections contributing to electron-nucleus interactions include the a) the anapole moment as well as b) terms that do not involve single photon exchange.

experimentally. The $\mathrm{E} 1$ transition is quite suppressed: the leading-order operator vanishes in a self-conjugate nucleus. (It corresponds to a translation of the center-of-mass.) The M1 is exceptionally strong, $\sim 10.3 \mathrm{~W} . \mathrm{u}$. Thus the second ratio is $\sim 110$. One concludes that the expected size of $P_{\gamma}$ is $\sim 10^{-3}$, four orders of magnitude above the typical scale of PNC in the NN system. Everything is known in Eq. (11) except the sign of the $M 1 / E 1$ ratio and the mixing matrix element.

Following early work by Barnes et al. [17, heroic efforts to measure $P_{\gamma}$ were made by the Queens [18, and Florence [19] groups, yielding $(8 \pm 39) \times 10^{-5}$. The DDH best-value prediction is $(208 \pm 49) \times 10^{-5}$. As the mixing is purely isovector, the expected enhancement due to neutral currents was not found.

First-principles calculations of PNC mixing matrix elements must address several difficulties. The underlying operator is dipole-like $\sim \vec{\sigma} \cdot \vec{p}$ and thus sensitive to spurious components, so that projection of the center-of-mass is important. As this operator couples opposite-parity shells, configurations in any included space are linked directly to the excluded space, leading to a sawtooth oscillation of the matrix element as new shells are added. The operator behaves under time reversal like the E1 operator, which is suppressed by correlations. $V_{P N C}$ is a surface operator, sensitive to the shapes of the single-particle wave functions. Most important, the down-side of exploiting parity doublets is the need to calculate a highly exclusive matrix element, one that exhausts a tiny fraction of the sum rule generated when $V_{P N C}$ operates on either member of the doublet.

In ${ }^{18} \mathrm{~F}$ these difficulties can be avoided by a simple trick: the doublet PNC mixing is identical, up to isospin rotation, to the exchange-current contribution to the axial-charge $\beta$ decay transition between the $0^{+} 1$ ground state of ${ }^{18} \mathrm{Ne}$ (the analog of the $0^{+} 1$ doublet state) and the $0^{-} 0$ member of the doublet. Furthermore the ratio of the exchange current contribution to the one-body operator $\vec{\sigma} \cdot \vec{p} \tau_{-}$is $\sim 1$ (both operators are of order $v / c$ ) and stable: the exchange current is effectively a renormalization of the one body operator. One can use the measured $\beta$ decay rate to determine the PNC mixing matrix element 20]. This argument, applied in a variety of nuclear structure calculations, leads to predictions of $\left\langle V_{P N C}\right\rangle$ that are stable to about $\pm 7 \%$. The case of ${ }^{19} \mathrm{~F}$ is similar, though there are additional uncertainties in this case because the mixing matrix element also contains an isoscalar piece.

Another constraint [21, 22] comes from atomic PNC experiments in which the nuclear anapole moment generates a dependence on the nuclear spin. The anapole moment is a weak radiative correction to the electron-nucleus interaction (see Fig. 4 that acts like a contact interaction and grows as $A^{2 / 3}$. In a heavy nucleus it can dominate the tree-level spin-dependent interaction from $\mathrm{V}(\mathrm{e})-\mathrm{A}$ (nucleus) $\mathrm{Z}$ exchange. There are various contributions to the anapole moment, but the most important term comes from nuclear ground-state polarization due to $V_{P N C}$. As the case of interest, ${ }^{133} \mathrm{Cs}[23$, has no ground-state parity doublet, the polarization is dominated by mixing with the collective giant resonance region.

A summary of what we have learned from experiment and theory is shown in Fig. 5 . To a good approximation the observables measured to date depend on two sets of couplings, one isoscalar $\sim-h_{\rho}^{0}-0.7 h_{\omega}^{0}$ and one isovector $\sim f_{\pi}-0.12 h_{\rho}^{1}-0.18 h_{\omega}^{1}$. (The constraint from $A_{L}^{\vec{p}+p}$ is plotted assuming the DDH value for $h_{\rho}^{2}$.) The overall consistency of the results is not high. It appears from $P_{\gamma}$ in ${ }^{18} \mathrm{~F}$ that the isovector coupling, where one expects the neutral current to dominate, is significantly smaller than the DDH best value. There is reasonable agreement between the odd-proton cases, ${ }^{19} \mathrm{~F}$ and $\vec{p}+\alpha$, which intersect with the ${ }^{18} \mathrm{~F}$ band at a point roughly consistent with the DDH best value for the isoscalar coupling. However, the odd-proton constraint from ${ }^{133} \mathrm{Cs}$ favors larger values of the couplings. (A bit of a very broad band from the upper bound on the ${ }^{205} \mathrm{Tl}$ anapole moment is also shown: the uncertainty in this result is such that it does not impact the conclusions drawn from the Fig. 5.) 


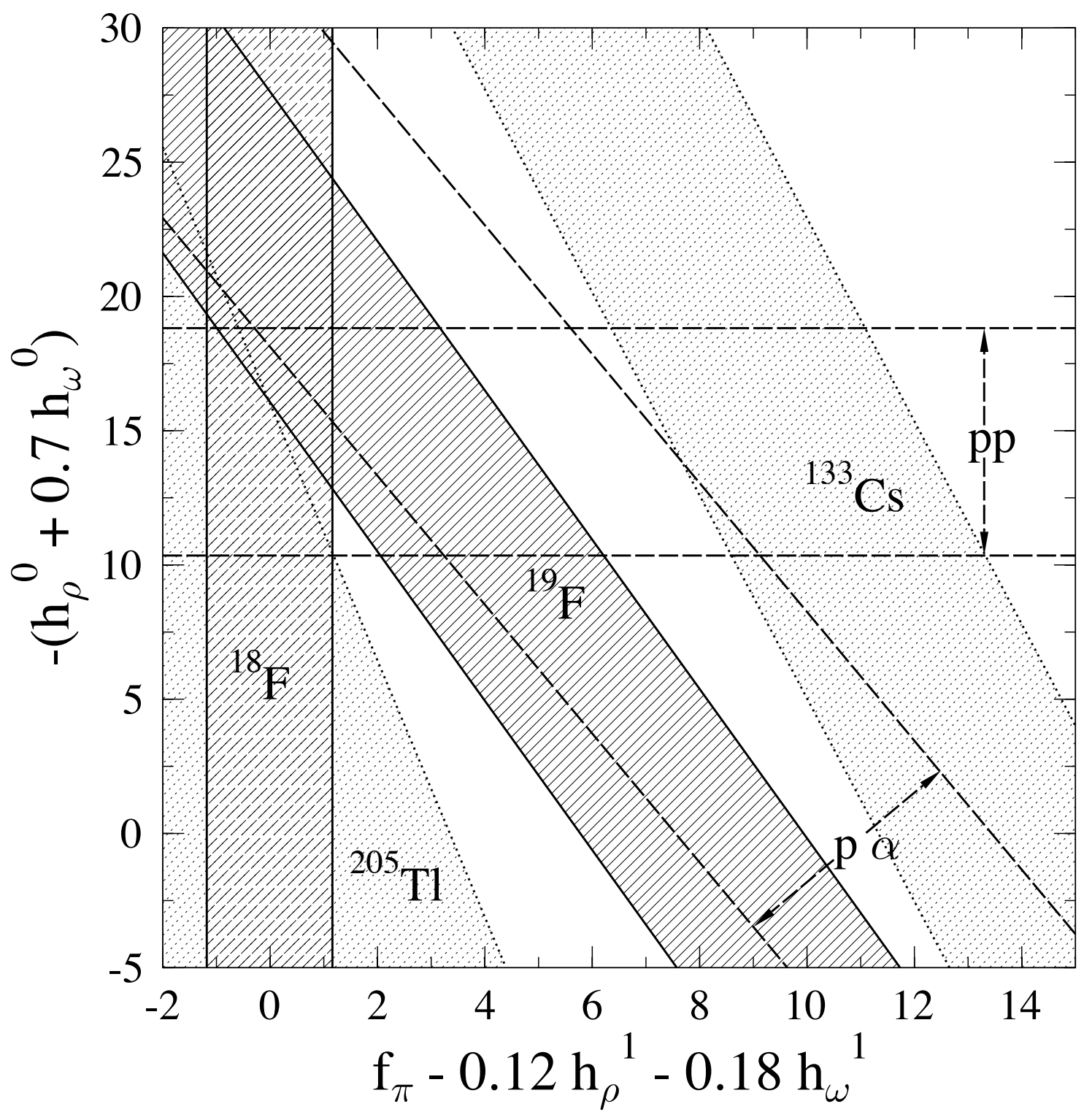

FIG. 5: Experimental PNC constraints as a function of the effective isoscalar and isovector couplings.

\section{SUMMARY}

The study of hadronic PNC has proven to be a very challenging area: both the experiments and the theoretical analysis are difficult. While some reasonable consistency exists between the ${ }^{18} \mathrm{~F},{ }^{19} \mathrm{~F}, \vec{p}+\alpha$, and $\vec{p}+p$ results (assuming $h_{\rho}^{2}$ is near the DDH best value), error bars are large and there is no significant redundancy among the measurements.

The conclusion from ${ }^{18} \mathrm{~F}$ that the isovector coupling is small, compared to the DDH best value, may be one of the more solid results. Several ${ }^{18} \mathrm{~F}$ experiments have placed tight upper bounds on $P_{\gamma}$, and the analysis, though it involves a complex nucleus, is unusually free of structure uncertainties. Consequently, we have yet to find evidence for neutral currents in $\Delta S=0$ interactions. Such suppression, relative to the isovector strength, is superficially reminiscent of the enhancements embodied in the $\Delta \mathrm{I}=1 / 2$ rule in flavor-changing reactions.

Clearly a lot remains to be done. The ongoing effort to measure $d \phi / d z$ in $\vec{n}+\alpha$ is important, as the comparison 
with $\vec{p}+{ }^{4} \mathrm{He}$ would allow an alternative $\Delta \mathrm{I}=0 / \Delta \mathrm{I}=1$ separation to be made, checking the pattern seen in Fig. 5 . The discrepancy involving the ${ }^{133} \mathrm{Cs}$ anapole moment is troublesome. As the control of systematics in that experiment required years of effort, it is not clear when the next anapole moment measurement will be made. But, from a theoretical perspective, such a measurement in an odd-neutron system would be useful in a PNC isospin analysis. If one could tighten the constraints on the isoscalar and isovector couplings, $A_{L}^{\vec{p}+p}$ would become an independent test of $h_{\rho}^{2}$.

While progress has been slow over the past decade, new facilities such as the SNS (with its high-intensity cold neutron beam) and FRIB (a possible source of radioactive nuclei with enhanced anapole moments) may help the field along in the next few years.

Good progress has been made in theory, with the development of a more systematic expansion for the effective PNC interaction being one recent example. But the lack of redundancy among experiments puts a lot of stress on theory, requiring one to make use of constraints in NN, few-body, and nuclear systems. It is not clear whether results from NN and few-body systems should be compared naively with those from complex nuclei. Couplings extracted from complex nuclei are necessarily effective, defined in the context of chosen shell-model spaces. We have many examples in nuclear physics (the axial-vector coupling $g_{A}$ being a celebrated one) where the shell-model coupling is not the underlying bare coupling. There has been some work comparing PNC calculations in small included spaces with those in larger ones. There is a significant dependence on the model space, indicating that effective couplings may differ substantially from the bare values. One good exercise for theorists might be to explore this question in a light nucleus where many shells can be included, in order to test the evolution of $\left\langle V_{P N C}\right\rangle$ with shell number. This could provide some guidance in interpreting results from systems like ${ }^{18} \mathrm{~F}$.

\section{Acknowledgments}

I thank C.-P. Liu for helpful discussions. This work was supported in part by the U.S. Department of Energy, Office of Nuclear Physics.

[1] E. G. Adelberger and W. C. Haxton, Ann. Rev. Nucl. Part. Sci. 35, 501 (1985).

[2] C.-P. Liu, nucl-th/0703008/, to appear in Proc. Second Meeting of the APS Topical Group On Hadronic Physics.

[3] G. S. Danilov, Phys. Lett. B 35, 579 (1971) and Phys. Lett. 18, 40 (1965).

[4] B. Desplanques, J. F. Donoghue, and B. R. Holstein, Ann. Phys. 124, 449 (1980).

[5] V. M. Dubovik and S. V. Zenkin, Ann. Phys. 172, 100 (1986).

[6] G. B. Feldman, G. A. Crawford, J. Dubach, and B. R. Holstein, Phys. Rev. C 43, 863 (1991).

[7] N. Kaiser and U. G. Meissner, Nucl. Phys. A 489, 671 (1988) and 499, 699 (1989).

[8] S. L. Zhu, C. M. Maekawa, B. R. Holstein, M. J. Ramsey-Musolf, and U. van Kolck, Nucl. Phys. A 748, 435 (2005).

[9] C.-P. Liu, Phys. Rev. C 75, 065501 (2007).

[10] P. D. Eversheim et al., Phys. Lett. B 256, 11 (1991).

[11] S. Kistryn et al., Phys. Rev. Lett. 58, 1616 (1987).

[12] V. A. Knyazkov et al., Nucl. Phys. A 197, 241 (1972).

[13] J. F. Cavaignac, B. Vignon, and R. Wilson, Phys. Lett. B 67, 148 (1977).

[14] D. M. Markoff, J. Res. Natl. Inst. Stand. Technol. 110, 209 (2005).

[15] J. Lang et al., Phys. Rev. Lett. 54, 170 (1985).

[16] A. Micherdzinska, http://www.int.washington.edu/talks/WorkShops/, 2007.

[17] C. A. Barnes et al., Phys. Rev. Lett. 40, 840 (1978).

[18] S. A. Page et al., Phys. Rev. C 35, 1119 (1987) and Phys. Rev. Lett. 55, 791 (1985).

[19] M. Bini et al., Phys. Rev. Lett. 55, 795 (1985).

[20] W. C. Haxton, Phys. Rev. Lett. 46, 698 (1981).

[21] W. C. Haxton, C. P. Liu, and M. J. Ramsey-Musolf, Phys. Rev. C 65, 045502 (2002) and Phys. Rev. Lett. 86, 5247 (2001).

[22] V. V. Flambaum and D. W. Murray, Phys. Rev. C 56, 1641 (1997).

[23] C. S. Wood et al., Science 275, 1759 (1997). 